Sección Agrícola / Agriculture

Artículo de investigación / Research paper

\title{
Nuevos registros de moscas de la fruta (Diptera: Tephritidae) en el Estado de Alagoas, Brasil
}

\author{
New records of fruit flies (Diptera: Tephritidae) in the Alagoas State, Brazil \\ SIMONE SILVA DA COSTA ${ }^{1}$, JAKELINE MARIA DOS SANTOS ${ }^{2}$, SÔNIA MARIA FORTI BROGLIO², \\ NIVIA DA SILVA DIAS-PINI ${ }^{4}$ y MARIUXI GÓMEZ-TORRES ${ }^{5}$
}

\begin{abstract}
${ }^{1}$ Agrónomo, Ph. D. en Protección de plantas, Universidade Federal de Alagoas, Maceió, Alagoas, Brasil, simone costa85@hotmail.com, https://orcid. org/0000-0002-5023-7191. ² Agrónomo, Ph. D. en Protección de plantas, Universidade Federal de Alagoas, Maceió, Alagoas, Brasil, jackbilu@hotmail.com. https://orcid.org/0000-0001-5935-8347. ${ }^{3}$ Agrónomo, Ph. D. en Entomología. Profesor Universidade Federal de Alagoas, Maceió, Alagoas, Brasil, soniamfbroglio@gmail.com, http://orcid.org/0000-0002-2223-732X. ${ }^{4}$ Biólogo, Ph. D. en Entomología. Investigador Embrapa Agroindústria Tropical, Fortaleza, Ceará, Brasil, nivia.dias@embrapa.br. https://orcid.org/0000-0002-3664-812X. ${ }^{5}$ Biólogo, Ph. D. en Entomología. Agrohumane Nordinsdustries S.A., Guayaquil, Ecuador, mgtorres@nordlystech.com, http://orcid.org/0000-0002-3344-8392.
\end{abstract}

Autor para correspondencia: Nivia da Silva Dias-Pini. Biólogo, Ph. D. en Entomología. Investigador Embrapa Agroindústria Tropical, Fortaleza, Ceará, Brasil,nivia.dias@embrapa. $b r$, https://orcid.org/0000-0002-3664-812X.

Citación sugerida / Suggested citation: COSTA, S. S.; SANTOS, J. M.; BROGLIO, S. M. F.; DIAS-PINI, N. S.; GÓMEZ-TORRES, M. 2019. Nuevos registros de moscas de la fruta (Diptera: Tephritidae) en el Estado de Alagoas, Brasil. Revista Colombiana de Entomología 45 (1): e7808 https://doi.org/10.25100/socolen. v45i1.7808

Recibido: 31-may-2017

Aceptado: 20-ago-2018

Publicado: 31-ago-2019

Revista Colombiana de Entomología

ISSN (Impreso): 0120-0488

ISSN (En línea): 2665-4385

http://revistacolombianaentomologia.univalle.edu.co/

Open access

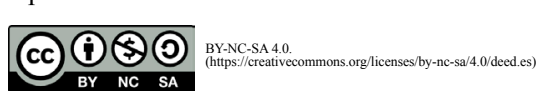

Publicadores / Publishers:

Sociedad Colombiana de Entomología

SOCOLEN (Bogotá, D. C., Colombia)

http://www.socolen.org.co

Universidad del Valle (Cali, Colombia)

http://www.univalle.edu.co/

(C) 2019 Sociedad Colombiana de Entomología

- SOCOLEN y Universidad del Valle - Univalle
Resumen: El presente trabajo propone como objetivo conocer la diversidad de moscas de las frutas, sus hospederos y parasitoides en los municipios del Estado de Alagoas, Brasil. En los meses de colecta se recolectaron un total de 17.394 especímenes de insectos frugívoros, de estos, 14.993 (86,2 \%) pertenecían al género Anastrepha (7.224 hembras y 7.769 machos) y $2.401(13,8 \%)$ a Ceratitis (1.196 hembras y 1.205 machos). En Maceió se recolectó una nueva especie que, hasta el momento, se la denomina como la morfo-especie Anastrepha sp. El mayor número de hembras pertenecían a Anastrepha obliqua la especie asociada al mayor número de plantas hospederas (ocho especies). De las 19 especies de frutales infestadas por moscas frugívoras, solo en nueve se registraron parasitoides. Del total de puparios de Anastrepha (26.724) emergieron 6.125 especímenes, 5.870 a pertenecían a la familia Braconidae, 169 a Pteromalidae, a 78 Figitidae y 8 a Torymidae. En este trabajo no se registró emergencia de parasitoides de los puparios de Ceratitis.

Palabras clave: Diversidad, avispas, neotrópico, manejo integrado, control.

Abstract: The aim of this study was to determine the diversity of fruit flies, their hosts and parasitoids in the State of Alagoas, Brazil. A total of 17,394 specimens of frugivore insects where recorded, of which 14,993 (86.2 \%) belonged to the genus Anastrepha (7,224 females and 7,769 males) and 2,401 (13.8\%) belonged to Ceratitis (1,196 females and 1,205 males). In Maceió, a new species of Anastrepha, which so far has been referred to as "morpho-species of Anastrepha sp.", has been collected. Besides showing the highest number of females collected during this study, Anastrepha obliqua was associated with the highest number of host species (eight species). Parasitoid emergence was associated with only 9 of the 19 fruit species infested with fruit flies. From all pupae of Anastrepha obtained in this study $(26,724)$, 6,125 parasitoid specimens have emerged, with 5,870 corresponding to Braconidae, 169 to Pteromalidae, 78 to Figitidae and 8 to Torymidae. In this study the emergence of parasitoids from puparia of Ceratitis was not recorded.

Keywords: Diversity, wasps, neotropic, integrated management, control.

\section{Introducción}

Las moscas de la fruta constituyen a nivel mundial, uno de los grupos más extensos de insectos fitófagos que ocasionan pérdidas económicamente importantes debido a los daños directos que ocasionan, ya sea por su característica de ovipositar en el epicarpio y mesocarpio de las frutas, así como en tejidos de las plantas o por el comportamiento de alimentación de las larvas que tornan inviable el consumo o uso industrial de las frutas. Los daños indirectos son el resultado de la infestación de microorganismos patógenos en los locales de la fruta atacados por estos insectos (Nava y Botton 2010). Además es importante resaltar las restricciones cuarentenarias por parte de los países donde la plaga no está registrada. El daño causado por moscas de la fruta afecta al rendimiento y los costos asociados con los servicios de vigilancia, control y erradicación, exigiendo una logística compleja (Raga 2005).

Muchos estudios se han desarrollado en Brasil con Tephritidae, sin embargo, en algunas regiones con potencial para la fruticultura, son pocos los trabajos que fomentan los levantamientos con moscas de la fruta (Canal et al. 1998). En el estado de Alagoas, el conocimiento de las especies de tefritídos es incipiente, siendo muy 
puntuales las informaciones que hace referencia a esta familia de dípteros. Los levatamientos poblacionales de este grupo se iniciaron en el 2000, obteniendo como resultando el registro de las especies Anastrepha fraterculus (Wiedemann, 1830), Anastrepha obliqua (Macquart, 1835) y Anastrepha sororcula Zucchi, 1979; así como de Ceratitis capitata (Wiedemann, 1824) (Gonçalves et al. 2006). Con relación a los parasitoides, Doryctobracon areolatus (Szépligeti, 1911) fue la única especie registrada. Sin embargo, la expansión de la fruticultura en el estado exige aumentar los estudios con moscas de la fruta hacia otras áreas de investigación para aportar informaciones relevantes sobre su diversidad y distribución en Brasil.

El muestreo de frutos tiene por objetivo medir la composición de especies asociadas al hospedero, así como evaluar el grado de infestación de la finca y el daño directo causado por las moscas de la fruta (Nascimento et al. 2000). También, es uno de los métodos más importantes para estudios con este grupo de insectos, pues además de generar informaciones sobre la diversidad de plantas hospederas, enemigos naturales y distribución geográfica, ofrece informaciones fundamentales para implementar el método más adecuado de manejo integrado de las especies plagas (Zucchi 2000b; Aluja et al. 2003).

Debido al incremento de las áreas dedicadas a la fruticultura en el Estado de Alagoas y de la importancia económica de las mosca de la fruta dentro de esta actividad agrícola, el presente trabajo propone como objetivo conocer la diversidad de esta plaga, sus hospederos y parasitoides en los municipios del Estado de Alagoas, Brasil.

\section{Materiales y métodos}

Este trabajo se desarrolló durante octubre 2010 hasta diciembre de 2011, en fincas comerciales y domésticas de las regiones de Agreste y al este del Estado de Alagoas, en los municipios de Anadia (9 $41^{\circ}$ '6"S, 36 18 '24'O, $163 \mathrm{~m}$ ), Arapi-

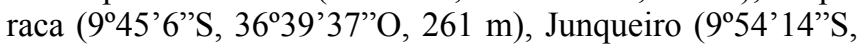
36²8'0'O, $214 \mathrm{~m}$ ), Maceió (9³9'59'S, 3544'6”O, $163 \mathrm{~m}$ ) y Palmeira dos Índios (9²4'58’S, 36³7'52”O, 196 m).

Recolecta de frutos. Para determinar la asociación entre la planta hospedera y la especie de moscas se realizaron reco- lectas sistemáticas de frutas. De esta manera, los muestreos fueron de forma aleatoria a diferentes alturas de las copas de los árboles, así como de frutos recién caídos en el suelo, que presentaran condiciones de conservación adecuadas y sin presentar orificios de salida de las larvas. Con relación al muestreo de las especiesde frutos, estos dependieron de la época del año y del período de fructificación de cada especie hospedera, así como de la disponibilidad de frutos.

A nivel del campo, los muestreos se realizaron sobre 25 especies fructíferas, distribuidas entre 14 familias botánicas: Anacardiaceae [Spondias sp., Spondias purpurea L., Spondias lutea L., Spondias dulcis Parkinson, Mangifera indica L., Anacardium occidentale L.], Annonaceae [Annona muricata], Apocynaceae [Hancornia speciosa], Cucurbitaceae [Momordica charantia L.], Euphorbiaceae [Manihot esculenta Crantz], Fabaceae [Inga sp.], Malpighiaceae [Malpighia glabra L.], Myrtaceae [Myrciaria trunciflora O. Berg., Psidium guajava L., Eugenia uniflora L., Sarracenia purpurea L., Psidium cattleianum Sabine, Syzygium cumini L.], Oxalidaceae [Averrhoa carambola L.], Passifloraceae [Passiflora edulis Sims], Rhamnaceae [Ziziphus joazeiro Mart.], Rubiaceae [Genipa americana L.], Rutaceae [Citrus sp., Citrus aurantium L.] y Sapotaceae [Manilkara zapota L.].

El material recolectado en campo fue transportado al laboratorio e inmediatamente acondicionado en bandejas plásticas, que contenían en el fondo una capa de arena tamizada y esterilizada como sustrato para las pupas. Después de la obtención de pupas, éstas fueron acondicionadas y separadas por género (Anastrepha y Ceratitis). Se conservaron los especímenes de moscas y/o parasitoides recolectados en alcohol al $70 \%$, para posterior identificación.

Identificación de los especímenes. La identificación de las especies de Anastrepha se realizó, siguiendo claves taxonómicas y con base al análisis del ápice del ovipositor de las hembras (Zucchi 2000a). Se contó además con la colaboración de los especialistas Roberto Antônio Zucchi (Departamento de Entomología y Acarología de la USP-ESALQ) y Keiko Uramoto (Departamento de Biología del Instituto de Biociencias, Universidad de São Paulo). Para la identificación de los especímenes de Ceratitis se utilizó el diagnósti-

Tabla 1. Especies de moscas frugívoras obtenidas de puparios de frutos muestreados en cinco municipios del Estado de Alagoas, Brasil. Octubre de 2010 a diciembre de 2011.

\begin{tabular}{|c|c|c|}
\hline Familia botánica & Especie hospedera & Especie frugívora /número de especimenes \\
\hline \multirow{5}{*}{ Anacardiaceae } & Spondias sp. & A. obliqua $(4,049) ;$ A. sororcula (1); A. fraterculus (1) \\
\hline & Spondias purpurea $\mathrm{L}$. & A. obliqua (477); A. sororcula (2); A. fraterculus (2); C. capitata (74) \\
\hline & Spondias lutea L. & $\begin{array}{l}\text { A. obliqua (733); A. sororcula (8); A. fraterculus (16); A. antunesi (3); } \\
\text { Anastrepha sp. (1); C. capitata (5) }\end{array}$ \\
\hline & Spondias dulcis Parkinson & A. obliqua (1) \\
\hline & Mangifera indica $\mathrm{L}$. & A. obliqua (13) \\
\hline Euphorbiaceae & Manihot esculenta Crantz & A. pickeli (339) \\
\hline Malpighiaceae & Malpighia glabra L. & A. sororcula (1); C. capitata (889) \\
\hline \multirow[t]{3}{*}{ Myrtaceae } & Psidium guajava $\mathrm{L}$. & $\begin{array}{l}\text { A. obliqua (3); A. sororcula (117); A. fraterculus (413); A. zenildae (3); } \\
\text { Anastrepha sp. (2); C. capitata (184) }\end{array}$ \\
\hline & Eugenia uniflora $\mathrm{L}$. & A. obliqua (52); A. sororcula (369); A. fraterculus (234); C. capitata (211) \\
\hline & Psidium cattleianum Sabine & A. fraterculus (30) \\
\hline Sapotaceae & Manilkara zapota L. & A. serpentina (6) \\
\hline Oxalidaceae & Averrhoa carambola $\mathrm{L}$. & A. obliqua (349); C. capitata (1.038) \\
\hline
\end{tabular}


co descrito por Foote (1980), basado en los rasgos morfológicos. No fue necesaria la confirmación de especímenes de $C$. capitata, ya que es la única especie del género que se encuentra en Brasil. En la identificación de las especies de parasitoides, se utilizaron las claves de Canal y Zucchi (2000). Los especímenes "voucher"permanecen depositados en el Laboratorio de Entomología de la Universidad Federal de Alagoas.

Parámetros evaluados. Se calculó el porcentaje de parasitismo siguiendo la ecuación: $\% \mathrm{P}=\left(\mathrm{n}^{\circ}\right.$ de parasitoides emergidos $/ n^{\circ}$ de moscas emergidas $+n^{\circ}$ de parasitoides emergidos) $\mathrm{x}$ 100. Los índices de infestación fueron calculados a partir de la división del número total de pupas por el total de frutos de cada especie recolectada (total pupas/total frutos) y el número total de pupas por el peso total $(\mathrm{kg})$ de cada especie recolectada (pupa/kg de fruto).

\section{Resultados y discusión}

Durante los 15 meses de trabajo, se recolectaron 17.394 especímenes de insectos frugívoros de éstos 14.993 (86,2 \%) pertencian al género Anastrepha (7.224 hembras y 7.769 machos) y $2.401(13,8 \%)$ a Ceratitis $(1.196$ hembras y 1.205 machos).

En los muestreos fueron identificadas nueve especies de tefritídos: Anastrepha antunesi Lima, 1938, Anastrepha fraterculus (Wiedemann, 1930), Anastrepha obliqua (Macquart, 1835), Anastrepha serpentina (Wiedemann, 1830), Anastrepha sororcula Zucchi, 1979, Anastrepha zenildae Zucchi, 1939, Anastrepha pickeli Lima, 1939, Anastrepha sp. y Ceratitis capitata (Wiedemann, 1824) (Tabla 1). Entre ellas: A. obliqua, $A$. fraterculus y $A$. sororcula han sido reportadas en el Estado de Alagoas (Gonçalves et al. 2006).

Tabla 2. Indice de infestación (puparios $/ \mathrm{kg}$ ) en frutos muestreados en cinco municipios del Estado de Alagoas, Brasil. Octubre de 2010 a diciembre de 2011.

\begin{tabular}{|c|c|c|c|}
\hline Especie vegetal & $\begin{array}{c}\mathrm{N}^{\circ} \text { de } \\
\text { puparios }\end{array}$ & Peso (kg) & $\begin{array}{l}\text { Índice de infestación } \\
\text { (puparios/kg de frutos) }\end{array}$ \\
\hline \multicolumn{4}{|l|}{ Anacardiaceae } \\
\hline Spondias lutea & 4.422 & 26,69 & 165,68 \\
\hline Spondias dulcis & 28 & 2,21 & 12,7 \\
\hline Mangifera indica & 148 & 30,46 & 4,9 \\
\hline Spondias purpurea & 2.646 & 30,12 & 87,84 \\
\hline Spondias sp. & 11.128 & 103,55 & 107,46 \\
\hline \multicolumn{4}{|l|}{ Euphorbiaceae } \\
\hline Manihot esculenta & 955 & 5,03 & 189,86 \\
\hline \multicolumn{4}{|l|}{ Malpighiaceae } \\
\hline Malpighia glabra & 920 & 84,62 & 10,87 \\
\hline \multicolumn{4}{|l|}{ Myrtaceae } \\
\hline Psidium guajava & 2.652 & 296,47 & 8,94 \\
\hline Eugenia uniflora & 3.575 & 29,64 & 120,61 \\
\hline Psidium cattleianum & 101 & 0,99 & 102,02 \\
\hline \multicolumn{4}{|l|}{ Oxalidaceae } \\
\hline Averrhoa carambola & 3.002 & 28,98 & 103,60 \\
\hline \multicolumn{4}{|l|}{ Sapotaceae } \\
\hline Manilkara zapota & 27 & 7,3 & 3,7 \\
\hline
\end{tabular}

Los especímenes de Anastrepha que no presentaron características morfológicas compatibles con las descritas en las claves de identificación y de las que no se obtuvo una confirmación taxonómica, hasta una posterior indentificación, fueron denominadas como una morfoespecie: Anastrepha sp.

Aunque se reportó una mayor diversidad de especies de Anastrepha (nueve), esta cantidad aún es considerada baja cuando se compara con las obtenidas en otros estados del nordeste. Los de Bahia, Maranhão, Piauí y Rio Grande do Norte, fueron los que presentaron mayor diversidad en la región con 31, 16, 16 y 13 especies registradas, respectivamente (Zucchi 2008).

Anastrepha obliqua estuvo asociada al mayor número de especies hospederas (ocho), con el mayor número de hembras recolectadas, habiendo emergido de frutos de Spondias sp., S. lutea, S. purpurea, S. dulcis, M. indica, P. guajava, E. uniflora y $A$. carambola. Esta es la única especie que se ha registrado en todos los estados brasileños, se caracteriza por presentar un hábito alimentar polífago, infestando alrededor de 35 especies de plantas, pertencientes a siete familias botánicas (Zucchi 2007). Sin embargo, se ha observado su marcada preferencia por anacardiáceas (Lima et al. 2007).

Anastrepha fraterculus fue reportada en seis especies hospederas: S. purpurea, S. lutea, P. guajava, E. uniflora, Spondias sp. y P. cattleianum. En Brasil, esta especie es considerada la más polífaga del género Anastrepha, así como, una de las más abundantes en las fincas del nordeste, sudeste (Nascimento et al. 1983; Martins et al. 2000; Souza-Filho et al. 2000) y del sur del país (Kovaleski et al. 1999; Garcia et al. 2003) y en este estudio, fue la segunda especie con mayor númerode hospederos registrados.

Se registraron especímenes de $C$. capitata sobre frutos de S. purpurea, S. lutea, P. guajava, E. uniflora, M. glabra y $A$. carambola. En el estado de Alagoas, esta especie fue observada infestando únicamente frutos de A. carambola (Gonçalves et al. 2006). En el sur de Bahia se reportó sobre frutos de Coffea arabica (Rubiaceae) y M. glabra (Melo et al. 2012). Entre los tefrítidos, C. capitata es considerada la especie más nociva, ya que ocasiona altos índices de daños económicos en la agricultura cuando comparada con otras moscas de la fruta, especialmente por ser la más cosmopolita y con mayor capacidad de dispersión (Malavasi 2009).

Anastrepha sororcula fue asociada a seis especies hospederas: Spondias sp., S. purpurea, S. lutea, P. guajava, E. uniflora y $M$. glabra. En las regiones semiáridas de Brasil, A. sororcula asume el status de plaga principal de $P$. guajava (Araújo y Zucchi 2003). La especie mencionada está más adaptada a las regiones de clima caliente y seco (Malavasi et al. 2000; Lemos et al. 2011) y se registra infestando frutos en diferentes hospederos principalmente sobre mirtáceas (Araújo et al. 2005; Raga 2005; Melo et al. 2012).

Anastrepha antunesi fue registrada únicamente sobre frutos de $S$. lutea y de acuerdo con otros autores, presenta preferencia por anacardiáceas (Santos 2015; Marsaro Junior et al. 2010; Melo et al. 2012). Sin embargo, hay informes de esta especie infestando mirtáceas en la región de Manaus, AM (Silva y Ronchi-Teles 2000). A. serpentina emergió apenas de frutos de la familia Sapotaceae (M. zapota). Melo et al. (2012) registran esta especie infestando frutos de Pouteria caimito, M. zapota (Sapotaceae) y Annonas quamosa (Annonaceae).

Los ejemplares de $A$. zenildae infestaron únicamente $P$. guajava. En el sudeste de Bahia esta especie también fue reportada apenas sobre mirtáceas (Silva et al. 2010); mientras que en la región sur, además infestó Oxalidaceae (Melo et 
Tabla 3. Especies de parasitoides asociados a mosca de las frutas y sus hospederos, así como el parasitismo natural registrado en cinco municipios del Estado de Alagoas, Brasil. Octubre de 2010 a diciembre de 2011.

\begin{tabular}{|c|c|c|c|}
\hline Plantas hospederas & Mosca de las frutas & Parasitoides & IP (\%) \\
\hline Averrhoa carambola & A. obliqua & D. areolatus, A. anastrephae & 3,7 \\
\hline Spondias purpurea & A. obliqua & D. areolatus & 16,6 \\
\hline Eugenia uniflora & A. fraterculus, A. sororcula, A. obliqua & D. areolatus, A. anastrephae, U. anastrephae, Opius spp., pteromálidos & 25,1 \\
\hline Psidium cattleianum & A. fraterculus & D. areolatus, pteromálidos & 18,8 \\
\hline Spondias lutea & A. obliqua & $\begin{array}{l}\text { D. areolatus, A. anastrephae, U. anastrephae, Opius spp., figítidos, } \\
\text { pteromálidos }\end{array}$ & 35,8 \\
\hline Psidium guajava & A. fraterculus & D. areolatus & 1,7 \\
\hline Spondias sp. & A. obliqua & D. areolatus, $A$. anastrephae, figítidos, pteromálidos & 28,5 \\
\hline Mangifera indica & A. obliqua & D. areolatus & 1,3 \\
\hline Manihot esculenta & A. pickeli & Opius spp. & 0,7 \\
\hline
\end{tabular}

al. 2012). En las regiones de los estados de Minas Gerais y Rio Grande do Norte se considera plaga para el cultivo de $P$. guajava (Canal et al. 1998; Araújo et al. 2008). A. pickeli fue asociada únicamente con frutos de M. esculenta. Esta especie juntamente com Anastrepha manihoti Lima, 1934 son fácilmente encontradas alimentándose del fruto y la parte superior de las ramas de $M$. esculenta (Schmitt 2002).

Con relación al índice de infestación, se constató que, de los 25 hospederos estudiados, 12 no presentaron infestación por las moscas de la fruta: A. occidentale, A. muricata, $H$. speciosa, $M$. charantia, Inga sp., $M$. trunciflora, S. cumini, $P$. edulis, Z. joazeiro, G. americana, Citrus sp. y C. aurantium. En todos los municipios se obtuvo un total de 29.604 puparios (26.724 de Anastrepha y 2.880 de Ceratitis). Los tefritidos infestaron con mayor intensidad $M$. esculenta, $S$. lutea y $E$. uniflora (Tabla 2).

Ceratites capitata infestó los frutos de S. lutea, S. purpurea, M. glabra, P. guajava, E. uniflora y A. carambola. $M$. glabra fue el único cultivo que registró niveles de infestación en todos los municipios. Barreto (2010) al recolectar frutos de M. glabra en el municipio de Parnaíba (Estado de Piauí) registró el $100 \%$ de infestación por $C$. capitata. En trabajos con levantamiento poblacional de mosca de las frutas, desarrollados por otros autores, también se observó que el cultivo de M. glabra presenta alta susceptibilidad a este tefrítido. Esto puede ser explicado, pues tanto la M. glabra como C. capitata son especies exóticas (Araújo et al. 2005; Feitosa et al. 2008; Sá et al. 2008; Alvarenga et al. 2009).

De las 19 especies frutíferas infestadas por moscas frugivoras, apenas en nueve se registraron parasitoides. Del total de puparios de Anastrepha (26.724) emergieron 6.125 especímenes de parasitoides, correspondiendo 5.870 a la familia Braconidae, 169 a Pteromalidae, 78 a Figitidae y 8 a Torymidae. En este trabajo no se registró emergencia de parasitoides de las pupas de Ceratitis (Tabla 3).

Las recolectas a nivel de campo permitieron identificar cuatro especies de bracónidos: Doryctobracon areolatus (Szépligeti, 1911), Utetes anastrephae (Viereck, 1913), Asobara anastrephae (Muesebeck, 1958) y Opius sp. Estas especies se caracterizan por presentar una amplia distribución geográfica, siendo registradas en diversos levantamientos de parasitoides de moscas de la fruta en Brasil (Canal y Zucchi 2000). Las especies más frecuentes fueron $A$. anastrephae y D. areolatus, que representaron $51,7 \%$ y $36,0 \%$ de los especímenes obtenidos, respectivamente.
En la mayoría de los trabajos con levantamientos de moscas de la fruta, asociados a parasitoides, el predominio es de D. areolatus. En la región de Mossoró/Assu-RN, D. areolatus representó el 96,6\% de los especimes, el 1,5\% a $U$. anastrephae y $A$. anastrephae fue registrado con el 1,9\% (Araújo y Zucchi 2002). En el Estado de São Paulo de los especímenes colectados, el 75,5\% y 3,8\% correpondieron a $D$. areolatus y A. anastrephae, respectivamente (Marinho et al. 2009). En la región sur de Bahia, D. areolatus constituyó el 55,7\% de los especimenes, $U$. anastrephae el $43,3 \%$ y el $0,46 \%$ apenas representó a $A$. anastrephae (Melo et al. 2012).

La recolecta de frutos además de ser un método específico para asociar las especies de moscas infestantes, también es fundamental para el asocio de las especies de parasitoides formado a partir de una relación tritrófica (planta-plaga-insecto benéfico). Todos los frutales que tuvieron larvas/pupas de moscas parasitadas, pueden ser asociadas por lo menos a una especie de parasitoide (Tabla 3).

En este trabajo, no fue posible asociar a los ejemplares de la familia Torymidae con ninguna especie de tefrítido a pesar que fueron obtenidos de puparios provenientes de $E$. uniflora; de las muestras recolectadas en campo emergieron tres especies de moscas de la fruta: A. fraterculus, $A$. obliqua y $A$. sororcula.

En este trabajo, D. areolatus estuvo asociado y a tres especies de moscas de la fruta: A. fraterculus, A. obliqua y $A$. sororcula en ocho frutales. Pirovani et al. (2010), en levantamientos realizados en Viçosa-MG, también registraron $D$. areolatus asociados a las mismas especies de tefrítidos en frutos de $S$. purpurea, $P$. cattleianum, E. uniflora y $A$. carambola.

Los frutos de E. uniflora y $S$. lutea presentaron mayor diversidad de parasitoides, siendo los de $S$. lutea asociados a todos los parasitoides registrados y los de E. uniflora únicamente asociados con figítidos. Carvalho et al. (2010) obtuvieron $D$. areolatus, A. anastrephae, $U$. anastrephae y Opius sp. en frutos de Spondias sp. en nueve municipios del Estado de Bahia.

El parasitismo natural registrado, varió de $0,7 \%$ a 35,8 $\%$ en los frutos de $M$. esculenta y $S$. lutea, respectivamente (Tabla 3).

Con excepción de los frutos de M. esculenta, en los que la infestación de moscas de la fruta aún no representa importancia económica para este cultivo, los mayores índices de parasitismo fueron observados en frutos de menor tama- 
ño de S. lutea $(35,8 \%)$, Spondias sp. $(28,5 \%)$, E. uniflora $(25,1 \%)$, arazá $(18,8 \%)$ y $S$. purpurea $(16,6 \%)$. Las larvas de moscas de la fruta son fácilmente parasitadas en frutos de menor tamaño, de pericarpio delgado y mesocarpio liso (Canal y Zucchi 2000). En frutos de mayor tamaño (M. indica, $P$. guajava y $A$. carambola) 3,7\% fue el mayor índice registrado. Este hecho se puede atribuir a la capacidad que tienen las larvas de moscas de las frutas de profundizar en las pulpas, lo que dificulta la acción de los parasitoides ( $\mathrm{Si}$ vinski et al. 1998).

\section{Conclusiones}

La amplia disponibilidad de plantas hospederas y la diversidad de especies de moscas de la fruta registradas en el estado de Alagoas, son factores a ser considerados debido al potencial para ocasionar daño en frutas y restringir el acceso a los mercados internacionales de productos vegetales que pueden hospedar estas especies.

\section{Literatura citada}

ALUJA, M.; RULL, J.; SIVINSKI, J.; NORRBOM, A. L.; WHARTON, R. A.; MACIAS-ORDÓNEZ, R.; DIAS-FLEISCHER, F.; LÓPEZ, M. 2003. Fruit flies of the genus Anastrepha (Diptera: Tephritidae) and associated native parasitoids (Hymenoptera) in the tropical rain forest biosphere reserve of Montes Azules, Chiapas, Mexico. Environmental Entomology 32 (6): 1377-1385. https://doi.org/10.1603/0046-225X-32.6.1377

ALVARENGA, C. D.; MATRANGOLO, C. A. R.; LOPES, G. N.; SILVA, M. A.; LOPES, E. N.; ALVES, D. A.; NASCIMENTO, A. S.; ZUCCHI, R. A. 2009. Moscas-das-frutas (Diptera: Tephritidae) e seus parasitóides em plantas hospedeiras de três municípios do Norte do Estado de Minas Gerais. Arquivos do Instituto Biológico 76 (2): 195-204.

ARAÚJO, E. L.; ZUCCHI, R. A. 2002. Parasitoides (Hymenoptera: Braconidae) de moscas-das-frutas (Diptera: Tephritidae) na região de Mossoró/Assu, Estado do Rio Grande do Norte. Arquivos do Instituto Biológico 69 (2): 65-68.

ARAÚJO, E. L.; ZUCCHI, R. A. 2003. Moscas-das-frutas (Diptera: Tephritidae) em goiaba (Psidium guajava L.), em Mossoró/ Assu, RN. Arquivos do Instituto Biológico 70: 73-77.

ARAÚJO, E. L.; MEDEIROS, M. K. M.; SILVA, V. E.; ZUCCHI, R. A. 2005. Moscas-das-frutas (Diptera: Tephritidae) no semiárido do Rio Grande do Norte: plantas hospedeiras e índices de infestação. Neotropical Entomology 34 (6): 889-894. https://doi. org/10.1590/S1519-566X2005000600003

ARAÚJO, E. L.; SILVA, R. K.; GUIMARÃES, J. A.; SILVA, J. G.; BITTENCOURT, M. A. L. 2008. Levantamento e flutuação populacional de moscas-das-frutas (Diptera: Tephritidae) em goiaba Psidium guajava L., no município de Russas (CE). Revista Caatinga 21 (1): 138-146.

BARRETO, N. T. R. 2010. Moscas-das-frutas (Diptera: Tephritidae) e seus parasitóides em goiaba e acerola nos tabuleiros litorâneos, Parnaíba, Piauí, Brasil. Dissertação em agronomia. Universidade Federal do Piauí. Terezina, Brasil. 88 p.

CANAL, N. A.; ZUCCHI, R. A. 2000. Parasitóides - Braconidae. pp. 119-126. En: Malavasi, A.; Zucchi, R. A. (Eds.). Moscas-dasfrutas de importância econômica no Brasil: conhecimento básico e aplicado. Editora Holos. São Paulo. Brasil. 327 p.

CANAL, N. A.; ALVARENGA, C. D.; ZUCCHI, R. A. 1998. Níveis de infestação de goiaba por Anastrepha zenildae Zucchi (Diptera: Tephritidae), em pomares comerciais do norte de Minas Gerais. Anais da Sociedade Entomológica do Brasil 27 (4): 657-661. https://doi.org/10.1590/S0301-80591998000400021

CARVALHO, R. da S.; SOARES FILHO, W. dos S.; RITZINGER, R. 2010. Umbu-cajá como repositório natural de para- sitoide nativo de moscas-das-frutas. Pesquisa Agropecuária Brasileira 45 (10): 1222-1225. https://doi.org/10.1590/S0100204X2010001000024

FEITOSA, S. S.; SILVA, P. R. R.; PÁDUA, L. E. M.; CARVALHO, E. M. S.; PAZ, J. K. S.; PAIVA, D. R. 2008. Flutuação populacional de moscas-das-frutas (Diptera: Tephritidae) associadas a variedades de manga no município de José de Freitas-Piauí. Revista Brasileira de Fruticultura 30 (1): 112-117. https://doi. org/10.1590/S0100-29452008000100021

FOOTE, R. H. 1980. Fruit fly genera South of the United States. Science and Education Administration, Washington. 79 p.

GARCIA, F. M.; CAMPOS, J. V.; CORSEUIL, E. 2003. Flutuação populacional de Anastrepha fraterculus (Wiedemann, 1830) (Diptera: Tephritidae) na Região oeste de Santa Catarina, Brasil. Revista Brasileira de Entomologia 47 (3): 415-420. https://doi. org/10.1590/S0085-56262003000300009

GONÇALVES, G. B.; SANTOS, J. C. G.; SILVA, C. E.; SANTOS, E. S. S.; NASCIMENTO, R. R.; SANT'ANA, A. E. G.; ZUCCHI, R. A. 2006. Occorrence of fruit flies (Diptera: Tephritidae) in the state of Alagoas, Brasil. Florida Entomologist 89 (1): 93-94. https://doi.org/10.1653/00154040(2006)89[93:OOFFDT]2.0.CO;2

KOVALESKI, A.; URAMOTO, K.; SUGAYAMA, R. L.; CANAL, D. N. A.; MALAVASI, A. 1999. A survey of Anastrepha Schiner (Diptera, Tephritidae) species in the apple growing area of the state of Rio Grande do Sul, Brazil. Revista Brasileira de Entomologia 43 (3/4): 229-234.

LEMOS, W. P.; ARAÚJO, S. C. A.; SILVA, R. A.; PEREIRA, J. D. B. 2011. Conhecimento sobre moscas-das-frutas no Estado do Pará. pp. 261-272. En: Silva, R. A; Lemos, W. P.; Zucchi, R. A. (Eds.). Moscas-dasfrutas na Amazônia brasileira: diversidade, hospedeiros e inimigos naturais. Embrapa Amapá. Macapá. Brasil. 299 p.

LIMA, J. C.; SANTOS, W.; CARVALHO, C. A. 2007. Moscas-dasfrutas (Diptera: Tephritidae) associadas ao umbu-cajá (Anacardiaceae) no vale do rio Paraguaçu, Bahia, Brasil. Revista Brasileira de Agrociência 13 (3): 399-402.

MALAVASI, A. 2009. Biologia, ciclo de vida, relação com o hospedeiro, especies importantes e bioecologia de tefritídeos. pp. 1-5. En: Malavasi, A.; Virgínio, J. (Eds.). Biologia, monitoramento e controle: V Curso Internacional de Capacitação em Moscas-dasFrutas. MoscaMed Brasil. Juazeiro. Brasil. 96 p.

MALAVASI, A.; ZUCCHI, R. A.; SUGAYAMA, R. L. 2000. Biogeografia. pp. 93-99. En: Malavasi, A.; Zucchi, R. A. (Eds.). Moscasdas-frutas de importância econômica no Brasil: conhecimento básico e aplicado. Editora Holos. São Paulo. Brasil. 327 p.

MARINHO, C. F; SOUZA-FILHO, M. F de; RAGA, A.; ZUCCHI, R. A. 2009. Parasitóides (Hymenoptera: Braconidae) de moscasdas-frutas (Diptera: Tephritidae) no Estado de São Paulo: plantas associadas e parasitismo. Neotropical Entomology 38 (3): 321 326. https://doi.org/10.1590/S1519-566X2009000300004

MARSARO JÚNIOR, A. L.; SILVA, R. A. da; SILVA, W. R. da; LIMA, C. R.; FLORES, A. S.; RONCHI-TELES, B. 2010. New records of Anastrepha (Diptera: Tephritidae), its hosts and parasitoids in the Serra do Tepequém, Roraima state, Brazil. Revista de Agricultura (Brasil) 85 (1): 15-19.

MARTINS, D. S.; URAMOTO, K.; MALAVASI, A. 2000. Espírito Santo. pp. 253-258. En: Malavasi, A.; Zucchi, R. A. (Eds.). Moscas-das-frutas de importância econômica no Brasil: conhecimento básico e aplicado. Editora Holos. São Paulo. Brasil. $327 \mathrm{p}$.

MELO, E. A. S. F.; SANTOS, E.; ALMEIDA, F. R.; ROCHA, R. B.; SANTOS, O. O.; STRIKIS, P. C.; BITTENCOURT, M. A. L. 2012. Hospedeiros, níveis de infestação e parasitoides de moscas frugívoras (Diptera: Tephritidae e Lonchaeidae) em municípios da região Sul da Bahia. Magistra 24 (número especial): 8-16.

NASCIMENTO, A. S.; ZUCCHI, R. A.; SILVEIRA NETO, S. 1983. Dinâmica populacionaldas moscas-das-frutas do gênero Anastrepha (Dip., Tephritidae) no Recôncavo Baiano. Análise Faunística. Pesquisa Agropecuária Brasileira 18 (4): 319-328. 
NASCIMENTO, A. S.; CARVALHO, R. S.; MALAVASI, A. 2000. Monitoramento populacional. pp. 109-112. En: Malavasi, A.; Zucchi, R. A. (Eds.). Moscas-das-frutas de importância econômica no Brasil: conhecimento básico e aplicado. Editora Holos. São Paulo. Brasil. 327 p.

NAVA, D. E.; BOTTON, M. 2010. Bioecologia e controle de Anastrepha fraterculus e Ceratitis capitata em pessegueiro. Pelotas: Embrapa Clima Temperado, 29 p. (Embrapa Clima Temperado. Documentos, 315). Pelotas. Disponible en: http://www.infoteca. cnptia.embrapa.br/handle/doc/888672 [Fecha revisión: 20 julio 2015].

PIROVANI, V. D.; MARTINS, D. S.; SOUZA, S. A. S.; URAMOTO, K.; FERREIRA, P. S. F. 2010. Moscas-das-frutas (Diptera: Tephritidae), seus parasitoides e hospedeiros em Viçosa, Zona da Mata Mineira. Arquivos do Instituto Biológico 77 (4): 727-733.

RAGA, A. 2005. Incidência, monitoramento e controle de moscasdas-frutas na citricultura paulista. Laranja 26 (2): 307-322.

SANTOS, J. M. dos. 2015. Relação entre parasitoides nativos de moscas-das-frutas e Diachasmimorpha longicaudata (Ashmead, 1905) (Hymenoptera: Braconidae) em pomares no município de Maceió-AL, Brasil. Tese em Proteção de Plantas. Universidade Federal de Alagoas. Rio Largo, Brasil. 127 p.

SÁ, R. F.; CASTELLANI, M. A.; NASCIMENTO, A. S. do; BRANDÃO, M. H da S. T.; SILVA, A. N. da; PÉREZ-MALUF, R. 2008. Índice de infestação e diversidade de moscas-das-frutas em hospedeiros exóticos e nativos no pólo de fruticultura de Anagé, BA. Bragantia 67 (2): 401-411. https://doi.org/10.1590/ S0006-87052008000200016

SILVA, N. M. da; RONCHI-TELES, B. 2000. Amapá, Amazonas, Pará, Rondônia e Roraima. pp. 203-209. En: Malavasi, A.; Zucchi, R. A. (Eds.). Moscas-das-frutas de importância econômica no Brasil: conhecimento básico e aplicado. Editora Holos. São Paulo. Brasil. 327 p.

SILVA, J. G.; DUTRA, V. S.; SANTOS, M. S.; SILVA, N. M. O.; VIDAL, D. B.; NINK, R. A.; GUIMARÃES, J. A.; ARAUJO, E. L. 2010. Diversity of Anastrepha spp. (Diptera: Tephritidae) and associated braconid parasitoids from native and exotic hosts in southeastern Bahia, Brazil. Environmental Entomology 39 (5): 1457-1465. https://doi.org/10.1603/EN10079

SCHMITT, A. T. 2002. Principais insetos praga da mandioca e seu controle. pp. 351-369. En: Arruda, M. P. (Coord.). Culturas de tuberosas amiláceas Latino Americanas. Vol. 2. Fundação Cargill. São Paulo. Brasil. 540 p.
SIVINSKI, J.; ALUJA, M.; HOLLER, T.; EITAM, A. 1998. Phenological comparison of who braconid parasitoids of the Caribbean fruit fly (Diptera: Tephritidae). Environmental Entomology 27 (2): 360-365. https://doi.org/10.1093/ee/27.2.360

SOUZA-FILHO, M. F. de; RAGA, A.; ZUCCHI, R. A. 2000. Moscas-das-frutas nos Estados brasileiros: São Paulo. pp. 277-283. En: Malavasi, A.; Zucchi, R. A. (Eds.). Moscas-das-frutas de importância econômica no Brasil: conhecimento básico e aplicado. Editora Holos. São Paulo. Brasil. 327 p.

ZUCCHI, R. A. 2000a. Taxonomia. pp. 13-24. En: Malavasi, A.; Zucchi, R. A. (Eds.). Moscas-das-frutas de importância econômica no Brasil: conhecimento básico e aplicado. Editora Holos. São Paulo. Brasil. 327 p.

ZUCCHI, R. A. 2000b. Especies de Anastrepha, sinonímias, plantas hospedeiras e parasitóides. pp. 41-48. En: Malavasi, A.; Zucchi, R. A. (Eds.). Moscas-das-frutas de importância econômica no Brasil: conhecimento básico e aplicado. Editora Holos. São Paulo. Brasil. 327 p.

ZUCCHI, R. A. 2007. Diversidad, distribución y hospederos del género Anastrepha en Brasil. pp. 77-100. En: Ortiz-Hernandéz, V. Moscas de la fruta en Latinoamérica (Diptera: Tephritidae): diversidad, biología y manejo. S y G Editores. Distrito Federal. México. 167 p.

ZUCCHI, R. A. 2008. Fruit flies in Brazil: Anastrepha species their host plants and parasitoids. Disponible en: www.lea.esalq.usp. br/anastrepha/[Fecha revisión: 14 marzo 2019].

\section{Origen y financiación}

Los autores agradecen a los fruticultores, que permitieron, en sus áreas cultivadas, el desarrollo de los trabajos de maestría de la primera autora, quien dió origen a este artículo; y agradecemos a la Fundação de Amparo à Pesquisa do Estado de Alagoas (FAPEAL) por el apoyo em la financiación de la investigación.

\section{Contribución de los autores}

Simone Silva da Costa y Jakeline Maria dos Santos, conducción de los experimentos y redacción del artículo; Mariuxi Gómez-Torres análisis de datos y redacción del artículo; Sonia Maria Forti Broglio y Nivia da Silva Dias-Pini planificación de la investigación y asesor de maestría de la primera autora. 